\title{
Genome-wide association study of cardiovascular disease in testicular cancer patients treated with platinum-based chemotherapy
}

\author{
Lars C. Steggink $\mathbb{D}^{1} \cdot$ Hink Boer ${ }^{1} \cdot$ Coby Meijer $^{1} \cdot$ Joop D. Lefrandt ${ }^{2} \cdot$ Leon W. M. M. Terstappen $\mathbb{1}^{3} \cdot$ \\ Rudolf S. N. Fehrmann ${ }^{1} \cdot$ Jourik A. Gietema (iD ${ }^{1}$
}

Received: 13 December 2019 / Revised: 4 September 2020 / Accepted: 23 September 2020 / Published online: 3 October 2020

(c) The Author(s) 2020. This article is published with open access

\begin{abstract}
Genetic variation may mediate the increased risk of cardiovascular disease (CVD) in chemotherapy-treated testicular cancer (TC) patients compared to the general population. Involved single nucleotide polymorphisms (SNPs) might differ from known CVD-associated SNPs in the general population. We performed an explorative genome-wide association study (GWAS) in TC patients. TC patients treated with platinum-based chemotherapy between 1977 and 2011, age $\leq 55$ years at diagnosis, and $\geq 3$ years relapse-free follow-up were genotyped. Association between SNPs and CVD occurrence during treatment or follow-up was analyzed. Data-driven Expression Prioritized Integration for Complex Trait (DEPICT) provided insight into enriched gene sets, i.e., biological themes. During a median follow-up of 11 years (range 3-37), CVD occurred in $53(14 \%)$ of 375 genotyped patients. Based on 179 SNPs associated at $p \leq 0.001,141$ independent genomic loci associated with CVD occurrence. Subsequent, DEPICT found ten biological themes, with the RAC2/RAC3 network (linked to endothelial activation) as the most prominent theme. Biology of this network was illustrated in a TC cohort $(n=60)$ by increased circulating endothelial cells during chemotherapy. In conclusion, the ten observed biological themes highlight possible pathways involved in CVD in chemotherapy-treated TC patients. Insight in the genetic susceptibility to CVD in TC patients can aid future intervention strategies.
\end{abstract}

\section{Introduction}

Testicular cancer (TC) is the most common malignancy in men between 20 and 40 years of age. Over $80 \%$ of patients with metastatic TC is cured with platinum-based chemotherapy [1]. Consequently, the number of TC survivors

Supplementary information The online version of this article (https:// doi.org/10.1038/s41397-020-00191-8) contains supplementary material, which is available to authorized users.

Jourik A. Gietema

j.a.gietema@umcg.nl

1 Department of Medical Oncology, University Medical Center Groningen, University of Groningen, Groningen, Hanzeplein 1, 9713 GZ Groningen, The Netherlands

2 Department of Internal Medicine, Division of Vascular Medicine, University Medical Center Groningen, University of Groningen, Groningen, Hanzeplein 1, 9713 GZ Groningen, The Netherlands

3 Medical Cell BioPhysics, University of Twente, Drienerlolaan 5, 7522 NB Enschede, The Netherlands steadily increases. High cure rates come at the trade-off of increased risk of cardiovascular disease (CVD), attributed to chemotherapy. Compared to age-matched controls, patients treated with bleomycin, etoposide, and cisplatin chemotherapy have a hazard ratio for coronary artery disease of 5.7 (95\% confidence interval, CI, 1.9-17.1), and for atherosclerotic disease of 4.7 (95\% CI, 1.8-12.2) [2]. Moreover, cardiovascular mortality is increased after chemotherapy for TC with a standardized mortality ratio of 1.36 (95\% CI, 1.03-1.78) [3]. In these relatively young men, the burden of cardiovascular morbidity and mortality due to chemotherapy is an important determinant of long-term outcome.

Genetic variation is thought to mediate in part the increased risk of CVD in chemotherapy-treated TC patients. However, involved single nucleotide polymorphisms (SNPs) might differ from the currently known SNPs associated with increased risk of CVD in the general population.

A limited number of studies reported on associations between SNPs and late complications of TC treatment. These studies mostly focused on SNPs in specific genes rather than using unbiased genome-wide approaches. SNPs 
in the glutathione S-transferase genes GSTP1 and GSTM3 have been associated with neurotoxicity and ototoxicity in platinum-treated TC patients [4-6]. SNPs in the 5- $\alpha$ reductase type II (SRD5A2) gene were associated with prevalence of the metabolic syndrome in TC survivors after platinum-based chemotherapy [7]. This finding, however, could not be replicated [8]. A recent study in 188 platinumtreated TC patients reported that a SNP in solute carrier gene SLC16A5 was associated with cisplatin-induced ototoxicity [9]. In the Platinum Study cohort of 511 TC survivors, a SNP in the Wolframin ER transmembrane glycoprotein (WFS1) was associated with ototoxicity [10].

To date, however, genetic variation has not been investigated in relation to CVD after TC treatment. As with the reported associations between SNPs and neurotoxicity, ototoxicity, and the metabolic syndrome, insight in genetic variation and biological pathways associated with cardiovascular toxicity may help to estimate the increased risk for CVD and to guide preventive cardiovascular intervention strategies in TC patients treated with platinum-based chemotherapy. We performed an exploratory genomewide association study (GWAS) in TC patients treated with platinum-based chemotherapy to gain insight into the SNPs underlying susceptibility to CVD in this population.

\section{Patients and methods}

\section{Patient selection and phenotype data}

Patients were selected from the institutional data-biobank on TC patients treated at the University Medical Center Groningen between 1977 and 2011. Inclusion criteria were (a) advanced seminoma or non-seminoma TC, Royal Marsden Hospital stage II, III, or IV, (b) treated with platinum-based chemotherapy, (c) age $\leq 55$ years at diagnosis, (d) $\geq 3$ years relapse-free follow-up after start of firstline chemotherapy, or after second-line chemotherapy if given for early relapse within 3 years after TC diagnosis, (e) no chemotherapy or malignancy prior to $\mathrm{TC}$, and (f) no CVD prior to TC.

The phenotype endpoint of the GWAS was the occurrence of a cardiovascular event during treatment or followup, defined as any (1) coronary disease (myocardial infarction, acute coronary syndrome), (2) cerebrovascular infarction or transient ischemic attack, (3) cardiomyopathy or heart failure, (4) thromboembolic event (deep venous thrombosis, pulmonary embolism, venous access portassociated), (5) peripheral artery disease, or (6) other cardiovascular events (e.g., intracerebral hemorrhage, cardiac arrhythmia, or cardiac valve regurgitation or stenosis). Follow-up data were available through several prospective TC studies and medical records. Follow-up was censored in case of late relapse (more than 3 years after chemotherapy), except for teratoma treated by surgery alone. In addition, follow-up was censored in case of second malignancy, except for non-melanoma skin cancer. Study protocols were approved by the medical ethical review committee of the University Medical Center Groningen (ethical protocol code 2006/041), performed in accordance with the Declaration of Helsinki and each participant gave written informed consent.

\section{Genotype data}

Germline DNA was isolated from blood using a standard phenol-chloroform method or using the NucleoSpin Blood XL column (Macherey-Nagel, BIOKÉ, Leiden, The Netherlands). SNP array was performed according to suppliers' protocol using an Illumina HumanCytoSNP-12 v2.1 BeadChip (Illumina, San Diego, California, US) covering 298,563 SNPs. SNPs were called using Illumina GenomeStudio v2011.1 (Genotyping v1.9.4; Illumina Genome Viewer v1.9.0) and then exported (PLINK Input Report Plug-in v2.1.3 for GenomeStudio Software).

\section{Genotyping quality analysis}

Quality analysis was performed using PLINK 1.07, filtering out (1) SNPs with a call rate $<95 \%$, (2) samples with a SNP call rate $<95 \%$, (3) samples with mismatch between reported and predicted sex, (4) SNPs that have minor allele frequency $<5 \%$, and (5) samples with significant deviation from the Hardy-Weinberg equilibrium $(p<0.0001)[11]$.

\section{Association analysis}

Association between SNPs and the occurrence of CVD was assessed in PLINK by chi-squared test using the $\max (\mathrm{T})$ permutation procedure with 25,000 permutations. SNPs were clumped into independently associated loci based on linkage disequilibrium, using an empirical $p \leq 0.001$ as a threshold for association of index SNPs (PLINK parameters: --clump-p1 0.001 --clump-p2 0.01 --clump-r2 0.20 --clump-kb 500).

\section{Annotation of associated SNPs: genes}

The loci found in the association analysis were annotated with genes known to be associated with these loci using the following three methods. First, we determined the nearest gene(s) for each SNP as reported in dbSNP build 150 . Second, based on a publicly available large-scale mapping of cis and trans expression quantitative trait loci (eQTLs) in blood we determined for each SNP if that SNP has been reported to affect the expression of any gene [12]. Third, the 
SNPs found in the association analysis were used as input for the Data-driven Expression Prioritized Integration for Complex Trait (DEPICT) framework [13]. At the core of DEPICT, genes are functionally characterized by their membership probabilities across 14,461 gene sets. DEPICT takes genes in loci associated with the input SNPs and uses the shared gene set memberships of those genes to prioritize genes that have similar predicted functions. Genes were reported by HGNC gene symbol if possible (retrieved using $\mathrm{R} /$ Bioconductor package biomaRt 2.32.1), or alternatively by Ensemble identifiers.

\section{Insight in associated SNPs: gene sets clustered into 'biological themes'}

Next, gene set enrichment analysis was performed to gain insight in the biology underlying the associations between SNPs and the occurrence of CVD. To this end, DEPICT takes genes in loci associated with the input SNPs, and tests which of its 14,461 gene sets are enriched in those genes, at a threshold of nominal $p<0.001$. Since DEPICT is based on reconstituted gene sets of known molecular pathways from various sources, overlap between the reconstituted gene sets can be expected. Therefore, the enriched gene sets were clustered into 'biological themes' using affinity propagation as described next.

The enriched gene sets were extracted from DEPICT resource file containing the gene-gene set matrix of $z$-scores for 19,987 genes. Next, pairwise Pearson correlation coefficients were computed between all enriched gene sets, and similar gene sets were clustered into biological themes using affinity propagation clustering (using $\mathrm{R}$ package apcluster 1.4.4) [14]. Affinity propagation finds exemplar gene sets within the input gene sets that are representative for each of the clusters, and names the clusters after their exemplar gene set. As stated by the authors of DEPICT, the reconstituted gene sets should be interpreted in light of the genes that are mapped to them, since their identifiers are simply carried over from the predefined gene sets used in the development of DEPICT [13]. For the biological themes, we addressed this issue by renaming the biological theme, if necessary, after examining the main genes within each biological theme (determined by the absolute weighted mean $z$-score for each gene in the biological theme, using the multiplicative inverse of the nominal $\mathrm{p}$ of each gene set as a weight for each $z$-score).

\section{Circulating endothelial cells during chemotherapy as indicator of endothelial activation}

As a measure of cancer treatment-induced endothelial activation, the number of circulating endothelial cells (CECs) were measured in 60 patients with metastatic TC

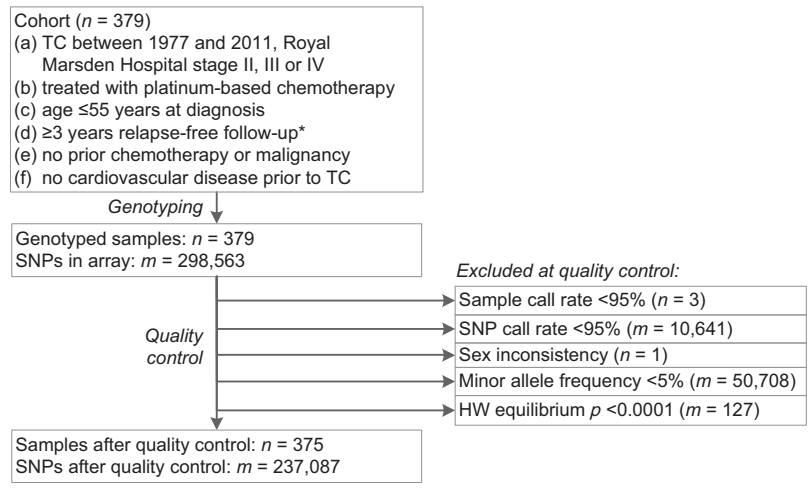

Fig. 1 Patient selection and quality control. * $\geq 3$ years relapse-free follow-up after start of first-line chemotherapy, or after second-line chemotherapy if given for early relapse within 3 years after TC diagnosis. HW equilibrium: Hardy-Weinberg equilibrium.

before and during three consecutive cycles of chemotherapy with bleomycin, etoposide, and cisplatin (BEP) using the CellSearch CEC Kit (Menarini Silicon Biosystems, Huntingdon Valley, PA, USA) according to the supplier's protocol [15]. In short, blood was collected in Cell Save Preservative tubes, and CECs were immunomagnetically enriched targeting CD146 followed by staining of the enriched cell population for CD45, CD105, and DAPI. The CD146 enriched cells were classified as CECs when CD105+/CD45-DAPI+ cells.

\section{Results}

Of the 379 genotyped patients, genotypes of 375 TC patients and 237,087 SNPs passed quality analysis (Fig. 1). All 375 TC patients had been treated with platinum-based chemotherapy between 1977 and 2011 (Table 1). Most chemotherapy regimens also contained bleomycin $(n=356$, 95\%). During a median follow-up of 11 years (range 3-37), CVD had occurred in 53 cases (14\%). These cases were median 4 years older at start of chemotherapy than patients who had no CVD $(p=0.009)$. At follow-up, cases met the criteria for metabolic syndrome more often $(67 \%$ versus $37 \%$ ) and used more antihypertensive and lipid lowering drugs than controls $(p<0.001$ for all).

In total, 179 SNPs were associated at $p \leq 0.001$ with the occurrence of any CVD during or after chemotherapy (Table S1). Clumping based on linkage disequilibrium resulted in 141 independent loci containing a total of 324 SNPs. These 141 loci and corresponding SNPs were annotated by finding nearest gene(s), cis or trans eQTLs, or DEPICT gene prioritization (Table 2). Since DEPICT only includes autosomal loci that do not overlap with the major histocompatibility complex region, gene prioritization resulted in 187 genes mapped to 129 loci. 
Table 1 Baseline patient and treatment characteristics and clinical follow-up data $(n=375)$.

\begin{tabular}{|c|c|c|c|c|c|}
\hline & \multicolumn{2}{|l|}{ Cases $(n=53)$} & \multicolumn{2}{|l|}{ Controls $(n=322)$} & \multirow[t]{2}{*}{$p$} \\
\hline & $\begin{array}{l}\text { Median (range) } \\
\text { or } n(\%)\end{array}$ & $\begin{array}{l}\text { Missing } \\
n(\%)\end{array}$ & $\begin{array}{l}\text { Median (range) } \\
\text { or } n(\%)\end{array}$ & Missing $n(\%)$ & \\
\hline Age at start chemotherapy (years) & $31(17-55)$ & - & $27(16-55)$ & - & 0.009 \\
\hline Age at follow-up (years) & $51(24-72)$ & - & $41(21-69)$ & - & $<0.001$ \\
\hline Follow-up duration (years) & $12(4-37)$ & - & $11(3-37)$ & - & 0.03 \\
\hline Royal Marsden Hospital stage & & - & & $3(1 \%)$ & 0.46 \\
\hline Stage II & $25(47 \%)$ & & $179(56 \%)$ & & \\
\hline Stage III & $6(11 \%)$ & & $31(10 \%)$ & & \\
\hline Stage IV & $22(42 \%)$ & & $109(34 \%)$ & & \\
\hline IGCCCG classification & & $2(4 \%)$ & & $3(1 \%)$ & 0.95 \\
\hline Good & $33(62 \%)$ & & $197(61 \%)$ & & \\
\hline Intermediate & $14(26 \%)$ & & $90(28 \%)$ & & \\
\hline Poor & $4(8 \%)$ & & $32(10 \%)$ & & \\
\hline Chemotherapy regime & & - & & - & - \\
\hline BEP followed by EP & $18(33 \%)$ & & $129(40 \%)$ & & \\
\hline BEP & $17(32 \%)$ & & $114(35 \%)$ & & \\
\hline PVB followed by PV maintenance & $3(6 \%)$ & & $16(5 \%)$ & & \\
\hline 2 PVB followed by 2 BEP & $1(2 \%)$ & & $16(5 \%)$ & & \\
\hline PVB & $4(8 \%)$ & & $11(3 \%)$ & & \\
\hline EP & $5(9 \%)$ & & $8(2 \%)$ & & \\
\hline CEB & 0 & & $10(3 \%)$ & & \\
\hline Other platinum-based chemotherapy & $5(9 \%)$ & & $18(6 \%)$ & & \\
\hline Any radiotherapy & $4(8 \%)$ & - & $4(1 \%)$ & - & - \\
\hline Abdominal & $2(4 \%)$ & & $2(0.6 \%)$ & & \\
\hline Cranial & 0 & & $2(0.6 \%)$ & & \\
\hline Thoracic & $1(2 \%)$ & & 0 & & \\
\hline Contralateral testicle & $1(2 \%)$ & & 0 & & \\
\hline No radiotherapy & $49(93 \%)$ & & $318(99 \%)$ & & \\
\hline Cardiovascular events & & - & & - & - \\
\hline Any event & $53(100 \%)$ & & 0 & & \\
\hline Cardiomyopathy $^{\mathrm{a}}$ & $6(11 \%)$ & & - & & \\
\hline Cerebrovascular $^{\mathrm{a}}$ & $8(15 \%)$ & & - & & \\
\hline Coronary $^{\mathrm{a}}$ & $19(36 \%)$ & & - & & \\
\hline Thromboembolic ${ }^{\mathrm{a}}$ & $19(36 \%)$ & & - & & \\
\hline Other $^{b}$ & $5(9 \%)$ & & - & & \\
\hline None & 0 & & $322(100 \%)$ & & \\
\hline Blood pressure at follow-up ( $\mathrm{mmHg}$ ) & & $3(6 \%)$ & & $26(8 \%)$ & \\
\hline Systolic & $139(110-190)$ & & $135(105-190)$ & & 0.07 \\
\hline Diastolic & $85(60-112)$ & & $80(50-124)$ & & 0.07 \\
\hline BMI at follow-up $\left(\mathrm{kg} / \mathrm{m}^{2}\right)$ & $26.4(19.4-40.6)$ & $5(9 \%)$ & $25.5(19.3-41.7)$ & $58(18 \%)$ & 0.04 \\
\hline Waist-hip ratio at follow-up & $1.0(0.9-1.3)$ & $8(15 \%)$ & $1.0(0.8-1.3)$ & $95(30 \%)$ & 0.60 \\
\hline Metabolic syndrome at follow-up & & $5(9 \%)$ & & $75(23 \%)$ & $<0.001$ \\
\hline Yes & $32(60 \%)$ & & $92(29 \%)$ & & \\
\hline No & $16(30 \%)$ & & $155(48 \%)$ & & \\
\hline Antihypertensive drugs at follow-up & & - & & $4(1 \%)$ & $<0.001$ \\
\hline Yes & $27(51 \%)$ & & $52(16 \%)$ & & \\
\hline No & $26(49 \%)$ & & $266(83 \%)$ & & \\
\hline
\end{tabular}


Table 1 (continued)

\begin{tabular}{|c|c|c|c|c|c|}
\hline & \multicolumn{2}{|l|}{ Cases $(n=53)$} & \multicolumn{2}{|c|}{ Controls $(n=322)$} & \multirow[t]{2}{*}{$p$} \\
\hline & $\begin{array}{l}\text { Median (range) } \\
\text { or } n(\%)\end{array}$ & $\begin{array}{l}\text { Missing } \\
n(\%)\end{array}$ & $\begin{array}{l}\text { Median (range) } \\
\text { or } n(\%)\end{array}$ & Missing $n(\%)$ & \\
\hline Lipid lowering drugs at follow-up & & - & & $3(1 \%)$ & $<0.001$ \\
\hline Yes & $21(40 \%)$ & & $27(8 \%)$ & & \\
\hline No & $32(60 \%)$ & & $292(91 \%)$ & & \\
\hline Antidiabetic drugs at follow-up & & - & & $3(1 \%)$ & 0.06 \\
\hline Yes & $4(8 \%)$ & & $7(2 \%)$ & & \\
\hline No & $49(93 \%)$ & & $312(97 \%)$ & & \\
\hline Testosterone suppletion at follow-up & & - & & $3(1 \%)$ & 0.75 \\
\hline Yes & $2(4 \%)$ & & $20(6 \%)$ & & \\
\hline No & $51(96 \%)$ & & $299(93 \%)$ & & \\
\hline Fasting glucose at follow-up (mmol/l) & $5.6(3.5-15.1)$ & $3(6 \%)$ & $5.4(1.3-9.4)$ & $104(32 \%)$ & 0.05 \\
\hline Total cholesterol at follow-up (mmol/l) & $4.6(3.1-7.6)$ & - & $5.2(2.8-9.7)$ & $5(2 \%)$ & $<0.001$ \\
\hline HDL cholesterol at follow-up (mmol/l) & $1.4(0.8-4.9)$ & $6(11 \%)$ & $1.2(0.3-5.6)$ & $56(17 \%)$ & 0.17 \\
\hline LDL cholesterol at follow-up (mmol/l) & $2.9(1.2-5.8)$ & $6(11 \%)$ & $3.3(0.4-6.5)$ & $57(17 \%)$ & 0.03 \\
\hline Triglyceride at follow-up (mmol/l) & $1.4(0.5-4.7)$ & - & $1.4(0.4-118)$ & $4(1 \%)$ & 0.90 \\
\hline Total testosterone at follow-up (nmol/l) & $13(4.7-33)$ & $6(11 \%)$ & $15(0.7-160)$ & $57(18 \%)$ & 0.01 \\
\hline eGFR at follow-up $\left(\mathrm{ml} / \mathrm{min} / 1.73 \mathrm{~m}^{2}\right)$ & $90(18-127)$ & - & $92(18-127)$ & $4(1 \%)$ & 0.02 \\
\hline $\begin{array}{l}\text { Albuminuria in } 24 \mathrm{~h} \text { urine at follow-up } \\
(\mathrm{mg} / 24 \mathrm{~h})\end{array}$ & $6(0.9-1,152)$ & $33(62 \%)$ & $8(0-5,850)$ & $158(49 \%)$ & - \\
\hline Chronic kidney disease stage at follow-up & & - & & $4(1 \%)$ & 0.01 \\
\hline Stage 4 & 0 & & $1(0.3 \%)$ & & \\
\hline Stage $3 B$ & $3(6 \%)$ & & $1(0.3 \%)$ & & \\
\hline Stage $3 \mathrm{~A}$ & $2(4 \%)$ & & $12(4 \%)$ & & \\
\hline Stage 2 & $26(49 \%)$ & & $130(40 \%)$ & & \\
\hline Stage 1 & $22(42 \%)$ & & $174(54 \%)$ & & \\
\hline
\end{tabular}

Characteristics of TC patients with and without cardiovascular event were compared with Fisher's exact test and Mann-Whitney U test after removal of missing values, with two-sided $p<0.05$ considered significant.

$B E P$ bleomycin, etoposide, cisplatin, $B M I$ body-mass index, $C E B$ carboplatin, etoposide, bleomycin, $e G F R$ estimated glomerular filtration rate, $E P$ etoposide, cisplatin, $H D L$ high density lipoprotein, IGCCCG International Germ Cell Cancer Collaborative Group, $L D L$ low-density lipoprotein, $P V$ cisplatin, vinblastine, $P V B$ cisplatin, vinblastine, bleomycin.

${ }^{a}$ Patients with multiple cardiovascular events were counted in multiple categories.

${ }^{\mathrm{b}}$ Intracerebral hemorrhage $(n=1)$, cardiac arrhythmia $(n=2)$, and cardiac valve regurgitation or stenosis $(n=2)$.

Next, gene set enrichment analysis was performed in DEPICT to gain insight in the biology underlying the associations between SNPs and CVD, resulting in 33 gene sets $(p<0.001$, Table S2). These 33 gene sets were subsequently clustered into ten distinct gene set clusters to highlight the biological themes that are underlying the associations between SNPs and CVD in chemotherapy-treated TC patients (Fig. 2 and Table S3). These biological themes included the RAC2/ RAC3 network, metabolism and adiposity, immune response, and caspase cascade/apoptosis.

Since the most enriched gene sets were the RAC2 and RAC3 subnetworks, clustered as the RAC2/RAC3 network, we explored a biological readout of this finding. RAC2 and RAC3 have been implicated in endothelial activation and dysfunction $[16,17]$. Therefore, as a measure of endothelial activation, the number of CECs were measured over time in 60 patients with metastatic TC treated with chemotherapy. A significant increase in CECs during three consecutive cycles of platinum-based chemotherapy was observed (Fig. 3).

\section{Discussion}

In this explorative GWAS using a contemporary strategy in TC patients treated with platinum-based chemotherapy, we determined which SNPs were associated with the occurrence of CVD after start of chemotherapy in these patients. 
Table 2 For the 141 identified candidate loci, related genes were found by proximity, cis or trans eQTLs, or by gene prioritization in DEPICT.

\begin{tabular}{|c|c|c|c|}
\hline Locus & SNPs & $p$ & Genes \\
\hline 1 & rs983098(*), rs1374038, rs10461655 & 0.00004 & PARP8(d) \\
\hline 2 & rs1352436(*) & 0.00004 & ENSG00000250546(d) \\
\hline 3 & $\begin{array}{l}\text { rs12692720(*), rs6432774, rs1528431, } \\
\text { rs6743187 }\end{array}$ & 0.00004 & RND3(n,d), LINC01920(d) \\
\hline 4 & $\begin{array}{l}\text { rs199635(*), rs852937, rs543827, } \\
\text { rs473757, rs6918162 }\end{array}$ & 0.00004 & LINC01626(n), LINC00472(d), OGFRL1(ce) \\
\hline 5 & rs34814294(*) & 0.00004 & $\begin{array}{l}\text { MMP28(n*,d), CCL5(d), HEATR9(d), RDM1 } \\
\text { (d), TAF15(d), LYZL6(d) }\end{array}$ \\
\hline 6 & rs3849324(*) & 0.00008 & MALL(n*,d), LINC00116(d), NPHP1(d) \\
\hline 7 & $\begin{array}{l}\text { rs6538046(*), rs } 10858436, \text { rs } 4503615 \\
\text { rs2406250, rs } 2406254\end{array}$ & 0.00008 & $\operatorname{MGAT} 4 \mathrm{C}\left(\mathrm{n}^{*}, \mathrm{~d}\right)$ \\
\hline 8 & $\begin{array}{l}\text { rs } 4755718(*), r s 7929359, \text { rs12273774, } \\
\text { rs } 4755689, \text { rs } 7929102, \text { rs } 10768008, \\
\text { rs7939586 }\end{array}$ & 0.00008 & KIAA1549L(n*,d), ENSG00000255207(d) \\
\hline 9 & $\begin{array}{l}\text { rs11874286(*), rs1025206, } \\
\text { rs16970618, rs12457667, rs8093155 }\end{array}$ & 0.00008 & LOC105372076(n\#), MIR924HG(d) \\
\hline 10 & rs6687976(*), & 0.00012 & LINC01676(d) \\
\hline 11 & rs2123269(*), rs2100346, rs6987013 & 0.00012 & MRPS28(n*,d), TPD52(d,ce) \\
\hline 12 & $\begin{array}{l}\text { rs7744306(*), rs9347666, rs9456798, } \\
\text { rs10945861 }\end{array}$ & 0.00012 & $\operatorname{PACRG}\left(\mathrm{n}^{*}, \mathrm{~d}\right), \operatorname{PARK} 2(\mathrm{n}, \mathrm{d})$ \\
\hline 13 & rs10950657(*), rs6968554, rs1476080 & 0.00012 & $\begin{array}{l}\text { AHR(n,d), LOC101927609(n\#), } \\
\text { ENSG00000237773(d\#), ENSG00000236318 } \\
(d \#)\end{array}$ \\
\hline 14 & rs9459964(*) & 0.00016 & LOC105378150(n*\#), ENSG00000232197(d\#) \\
\hline 15 & rs4466027(*), rs7673254, rs13121254 & 0.00016 & LINC02261(n*), STIM2(d) \\
\hline 16 & rs6988639(*), rs1433393, rs2656118 & 0.00016 & SNTB1(d), HAS2(d) \\
\hline 17 & rs12439991(*), rs7163517, rs11857756 & 0.00016 & LOC105370777(n*), ENSG00000259450(d) \\
\hline 18 & rs10932020(*), rs7591187 & 0.00016 & $\mathrm{CD} 28(\mathrm{~d}, \mathrm{ce})$ \\
\hline 19 & rs6813846(*), rs9997501, rs892836 & 0.0002 & $\operatorname{STOX} 2(n *, d)$ \\
\hline 20 & $\begin{array}{l}\text { rs7748814(*), rs7742883, rs6914805, } \\
\text { rs6459467 }\end{array}$ & 0.0002 & $\operatorname{GMPR}(\mathrm{d}, \mathrm{ce}), \operatorname{ATXN1}(\mathrm{d}, \mathrm{ce})$ \\
\hline 21 & rs $2331545(*)$ & 0.0002 & $\operatorname{OVAAL}\left(\mathrm{n}^{*}, \mathrm{~d}\right)$ \\
\hline 22 & rs676740(*) & 0.0002 & AFDN(n*,d), ENSG00000235994(d) \\
\hline 23 & rs1263635(*), rs943888 & 0.00024 & TRAC(d) \\
\hline 24 & $\begin{array}{l}\text { rs } 755535(*), \text { rs } 12108497, \text { rs } 2130392, \\
\text { rs4069938 }\end{array}$ & 0.00024 & $\begin{array}{l}\text { PRIMPOL(n,d,ce), CENPU(n,d,ce), ACSL1 } \\
\text { (n), CASP3(d,ce) }\end{array}$ \\
\hline 25 & rs11164896(*), rs2783499 & 0.00024 & $\begin{array}{l}\text { CCDC18(n,d,ce), DR1(d,ce), TMED5(d,ce), } \\
\text { MTF2(d), FNBP1L(d), CCDC18-AS1(d) }\end{array}$ \\
\hline 26 & rs9324446(*), rs3887806, rs7837472 & 0.00024 & FAM135B(d) \\
\hline 27 & rs3934720(*) & 0.00024 & EIF2B5(d) \\
\hline 28 & rs $7702793(*)$ & 0.00024 & LOC105379160(n*\#), GRAMD3(d) \\
\hline 29 & rs10034996(*) & 0.00024 & ENSG00000251199(d) \\
\hline 30 & rs1826613(*), rs1227842 & 0.00024 & DLG2(n*,d) \\
\hline 31 & $\begin{array}{l}\text { rs } 4757245\left(^{*}\right), \text { rs } 4756786, \text { rs } 2970335, \\
\text { rs11023194, rs11023197, rs6486191, } \\
\text { rs11023210, rs } 3923294, \text { rs12295888, } \\
\text { rs11023223, rs2575825, rs10832275 }\end{array}$ & 0.00028 & $\begin{array}{l}\text { SPON1(n,d), PDE3B(d), RRAS2(d), COPB1 } \\
\text { (d,ce) }\end{array}$ \\
\hline 32 & rs1387092(*), rs1488745, rs6802020 & 0.00028 & CNTN4(d) \\
\hline 33 & rs17790008(*), rs6584652 & 0.00032 & $\operatorname{SORCS} 3\left(\mathrm{n}^{*}, \mathrm{~d}\right)$ \\
\hline 34 & rs10828065(*), rs12355916 & 0.00032 & $\operatorname{PLXDC} 2(\mathrm{~d})$ \\
\hline 35 & rs596557(*) & 0.00032 & TMX3(d) \\
\hline
\end{tabular}


Table 2 (continued)

\begin{tabular}{|c|c|c|c|}
\hline Locus & SNPs & $p$ & Genes \\
\hline 36 & rs $17377955(*)$ & 0.00032 & $\operatorname{DGKB}(\mathrm{d})$ \\
\hline 37 & rs11582429(*), rs2168951 & 0.00032 & LINC01732(d) \\
\hline 38 & $\begin{array}{l}\text { rs } 9949956(*), \text { rs } 8095771, \text { rs12966370, } \\
\text { rs } 7236288, \text { rs } 10871565\end{array}$ & 0.00032 & LINC01029(n), GALR1(d) \\
\hline 39 & rs6816525(*) & 0.00032 & $\operatorname{IRF} 2\left(\mathrm{n}^{*}, \mathrm{~d}\right)$ \\
\hline 40 & rs4896501(*), rs7753475 & 0.00032 & CLVS2(d) \\
\hline 41 & rs $2554728(*)$ & 0.00032 & $\operatorname{CSMD} 1\left(\mathrm{n}^{*}, \mathrm{~d}\right)$ \\
\hline 42 & rs10503759(*), rs969456 & 0.00032 & $\begin{array}{l}\text { LOC101929294(n*\#), ADAM7(d), } \\
\text { ENSG00000253643(d\#), ADAMDEC1(ce) }\end{array}$ \\
\hline 43 & rs4858795(*), rs6794875 & 0.00032 & $\begin{array}{l}\text { SHISA5(n*,d), PLXNB1(n,d), CCDC51(d), } \\
\text { FBXW12(d), PFKFB4(d,ce), TREX1(ce), } \\
\text { ATRIP(ce), NME6(ce), NCKIPSD(ce) }\end{array}$ \\
\hline 44 & rs4662553(*) & 0.00036 & LRP1B(d) \\
\hline 45 & rs17756443(*), rs16959991 & 0.00036 & $\mathrm{CDH} 13(\mathrm{n} *, \mathrm{~d})$ \\
\hline 46 & rs5931289(*), rs5929883, rs5931353 & 0.00036 & - \\
\hline 47 & rs12920637(*), rs7198542 & 0.00036 & $\mathrm{CDH} 13(\mathrm{n} *, \mathrm{~d})$ \\
\hline 48 & rs7745485(*), rs12524966, rs12198618 & 0.00036 & LOC105374974(n\#), HDGFL1(d) \\
\hline 49 & rs6692(*), rs9555784 & 0.0004 & $\operatorname{ARHGEF7}\left(\mathrm{n}^{*}, \mathrm{~d}, \mathrm{ce}\right)$ \\
\hline 50 & rs6467607(*) & 0.0004 & SLC13A4(d) \\
\hline 51 & rs10902531(*) & 0.0004 & SFSWAP(d) \\
\hline 52 & rs2215375(*) & 0.0004 & SPP2(d) \\
\hline 53 & rs7101204(*) & 0.0004 & $\operatorname{SVIL}\left(\mathrm{n}^{*}, \mathrm{~d}\right)$ \\
\hline 54 & rs $1555145(*)$ & 0.0004 & BTBD3(d) \\
\hline 55 & rs13243936(*) & 0.0004 & EPDR1(d), NME8(ce), GPR141(ce) \\
\hline 56 & $\begin{array}{l}\text { rs } 11772261(*), \text { rs11770352, } \\
\text { rs6462776, rs4723679, rs6462780 }\end{array}$ & 0.0004 & EPDR1(d) \\
\hline 57 & rs4432837(*), rs6864394 & 0.0004 & RGS7BP(n*,d) \\
\hline 58 & $\begin{array}{l}\text { rs11736162(*), rs1439381, rs1439382, } \\
\text { rs7684647 }\end{array}$ & 0.00044 & GUF1(d,ce), GNPDA2(ce) \\
\hline 59 & rs9459963(*), rs9366130, rs4540249 & 0.00044 & $\begin{array}{l}\text { ENSG00000232197(d), DLL1(ce), FAM120B } \\
\text { (ce) }\end{array}$ \\
\hline 60 & rs4676617(*) & 0.00044 & $\begin{array}{l}\text { LOC102724104(n*\#), CX3CR1(d,ce), } \\
\text { WDR48(ce) }\end{array}$ \\
\hline 61 & rs11025878(*), rs4644637, rs7941875 & 0.00044 & $\operatorname{NELL1}\left(\mathrm{n}^{*}, \mathrm{~d}\right)$ \\
\hline 62 & rs2040664(*) & 0.00044 & DNAH11(n*,d) \\
\hline 63 & $\begin{array}{l}\text { rs11066610(*), rs16942882, } \\
\text { rs11066638 }\end{array}$ & 0.00044 & LHX5(d), LINC01234(d) \\
\hline 64 & rs1366906(*), rs6485532 & 0.00048 & CD82(d) \\
\hline 65 & rs3866223(*), rs11241999 & 0.00048 & $\operatorname{ADAMTS19}\left(\mathrm{n}^{*}, \mathrm{~d}\right)$ \\
\hline 66 & rs2275696(*), rs3892248 & 0.00048 & NFASC(n*,d), DSTYK(ce) \\
\hline 67 & $\begin{array}{l}\text { rs12688573(*), rs5936239, rs2341921, } \\
\text { rs758439 }\end{array}$ & 0.00048 & $\operatorname{AFF} 2(n *)$ \\
\hline 68 & rs2027469(*) & 0.00048 & CRP(d), DUSP23(ce) \\
\hline 69 & $\begin{array}{l}\text { rs11800877(*), rs4657327, rs1415439, } \\
\text { rs12076657 }\end{array}$ & 0.00048 & $\mathrm{PBX} 1(\mathrm{~d})$ \\
\hline 70 & rs2070584(*) & 0.00052 & TIMP1(n*) \\
\hline 71 & rs28890299(*) & 0.00052 & LIPI(n*) \\
\hline 72 & rs11643432(*), rs10514583 & 0.00052 & $\mathrm{CDH} 13\left(\mathrm{n}^{*}, \mathrm{~d}\right)$ \\
\hline 73 & $\begin{array}{l}\text { rs } 4237648(*), \text { rs } 10742717, \text { rs } 4237647 \\
\text { rs1665150 }\end{array}$ & 0.00052 & TSPAN18(n,d) \\
\hline
\end{tabular}


Table 2 (continued)

\begin{tabular}{|c|c|c|c|}
\hline Locus & SNPs & $p$ & Genes \\
\hline 74 & $\begin{array}{l}\text { rs4689203(*), rs13123841, } \\
\text { rs10937629, rs13121492 }\end{array}$ & 0.00056 & $\operatorname{STK} 32 \mathrm{~B}(\mathrm{n} *, \mathrm{~d})$ \\
\hline 75 & rs9530423(*), rs1359500, rs9573531 & 0.00056 & TBC1D4(n*,d) \\
\hline 76 & rs7143719(*), & 0.00056 & $\operatorname{TSHR}\left(\mathrm{n}^{*}, \mathrm{~d}\right)$ \\
\hline 77 & $\begin{array}{l}\text { rs } 10822863(*), \text { rs2894011, rs2894015, } \\
\text { rs4143863 }\end{array}$ & 0.00056 & CTNNA3(n*,d) \\
\hline 78 & rs7610664(*), rs7609933 & 0.00056 & FGF12(n*,d) \\
\hline 79 & rs6558831(*), rs12680491, rs11136689 & 0.00056 & $\operatorname{CSMD} 1\left(\mathrm{n}^{*}, \mathrm{~d}\right)$ \\
\hline 80 & $\begin{array}{l}\text { rs } 4751878(*), \text { rs4752666, rs10887101, } \\
\text { rs6585804, rs11592039 }\end{array}$ & 0.00056 & $\operatorname{TACC} 2\left(\mathrm{n}^{*}, \mathrm{~d}\right)$ \\
\hline 81 & rs12965155(*) & 0.00056 & MIR924HG(d) \\
\hline 82 & rs760150(*) & 0.00056 & PCP4(n*,d), TMPRSS3(d) \\
\hline 83 & rs239953(*) & 0.00056 & $\operatorname{POR}\left(\mathrm{n}^{*}, \mathrm{~d}, \mathrm{ce}\right), \mathrm{RHBDD} 2(\mathrm{~d}, \mathrm{ce})$ \\
\hline 84 & rs10182928(*) & 0.00056 & SATB2(d), SATB2-AS1(d) \\
\hline 85 & rs617459(*), rs657426 & 0.0006 & $\operatorname{SETBP} 1(n *, d)$ \\
\hline 86 & rs12165104(*), rs12954590 & 0.0006 & TNFRSF11A(n*,d), ZCCHC2(d) \\
\hline 87 & rs11688528(*) & 0.00064 & LOC100506474(n*\#), TRIB2(d) \\
\hline 88 & rs6966799(*) & 0.00064 & $\operatorname{HDAC} 9(\mathrm{~d})$ \\
\hline 89 & rs6759648(*), rs7593846, rs9941639 & 0.00064 & LINC01798(n*), MEIS1(d) \\
\hline 90 & rs2973419(*) & 0.00064 & PRR16(d) \\
\hline 91 & $\begin{array}{l}\text { rs10456118(*), rs10948172, rs857601, } \\
\text { rs3799977, rs4714828, rs 10948197, } \\
\text { rs6919813 }\end{array}$ & 0.00064 & SUPT3H(n,d,ce), RUNX2(d) \\
\hline 92 & rs4795934(*), rs990510, rs12944367 & 0.00064 & TMEM132E(d) \\
\hline 93 & rs7831168(*), rs13269649, rs907991 & 0.00068 & FAM135B(d) \\
\hline 94 & rs9880546(*) & 0.00068 & LINC00578(n*), TBL1XR1(d) \\
\hline 95 & $\begin{array}{l}\text { rs1375547(*), rs9861237, rs9822731, } \\
\text { rs12498010, rs9880919 }\end{array}$ & 0.00068 & $\mathrm{CADM} 2(\mathrm{n} *, \mathrm{~d})$ \\
\hline 96 & $\begin{array}{l}\text { rs6557678(*), rs6988938, rs7824718, } \\
\text { rs7009973, rs7008867 }\end{array}$ & 0.00068 & $\begin{array}{l}\text { SLC25A37(d,ce), ENTPD4(ce), AC051642.5 } \\
\text { (ce\#) }\end{array}$ \\
\hline 97 & rs16993897(*) & 0.00068 & VAV1(d), ADGRE1(ce) \\
\hline 98 & rs16999330(*), rs4434196 & 0.00068 & $\operatorname{FSTL} 5\left(n^{*}, \mathrm{~d}\right)$ \\
\hline 99 & rs16880318(*), rs16880352 & 0.00072 & $\mathrm{KCNV} 1(\mathrm{~d})$ \\
\hline 100 & rs4947522(*), rs28633916, rs9642409 & 0.00072 & $\operatorname{COBL}(\mathrm{d})$ \\
\hline 101 & rs17078840(*) & 0.00072 & LINC00327(d) \\
\hline 102 & rs $40566(*)$ & 0.00072 & C5orf67(n*), MAP3K1(d) \\
\hline 103 & rs4528743(*) & 0.00072 & SLC16A14(n*,d) \\
\hline 104 & rs13249135(*) & 0.00072 & MIR2052HG(n*,d) \\
\hline 105 & rs747925(*), rs11236683 & 0.00072 & LOC105369395(n*\#), THAP12(d) \\
\hline 106 & rs10505371(*), rs17803964 & 0.00076 & ENPP2(n*,d), TAF2(ce) \\
\hline 107 & rs3911618(*) & 0.00076 & RGS7(n*,d) \\
\hline 108 & rs7691972(*) & 0.00076 & $\operatorname{ACSL1}\left(n^{*}, \mathrm{~d}\right), \operatorname{CASP} 3(\mathrm{ce})$ \\
\hline 109 & rs6507498(*), rs9807753, rs8093542 & 0.00076 & CABLES1(d) \\
\hline 110 & rs7722584(*), rs11948927 & 0.00076 & NLN(d) \\
\hline 111 & rs3812278(*), rs10255837 & 0.00076 & CNOT4(n*,d), NUP205(d) \\
\hline 112 & rs6773957(*), rs6444175 & 0.0008 & ADIPOQ(n*,d), LYST(te) \\
\hline 113 & $\begin{array}{l}\text { rs10858680(*), rs10777082, } \\
\text { rs11104704, rs11104713 }\end{array}$ & 0.0008 & $\mathrm{C} 12 \operatorname{orf50}(\mathrm{n} * \mathrm{~d})$ \\
\hline 114 & rs4577099(*) & 0.0008 & LOC102724084(n*\#), DYNLRB2(d) \\
\hline
\end{tabular}


Table 2 (continued)

\begin{tabular}{|c|c|c|c|}
\hline Locus & SNPs & $p$ & Genes \\
\hline 115 & rs8030490(*) & 0.0008 & AKAP13(n*,d,ce) \\
\hline 116 & rs11610234(*), rs4334084 & 0.0008 & TMEM132B(n*,d) \\
\hline 117 & rs4762060(*) & 0.0008 & KRT80(n*,d), C12orf80(d) \\
\hline 118 & rs6673313(*) & 0.0008 & LOC105378764(n*\#), NFIA(d) \\
\hline 119 & rs $2506145(*)$ & 0.00084 & $\operatorname{NRP} 1\left(n^{*}, \mathrm{~d}\right)$ \\
\hline 120 & rs898918(*), rs12100703 & 0.00084 & LINC01550(d) \\
\hline 121 & $\begin{array}{l}\text { rs4607409(*), rs300121, rs777573, } \\
\text { rs9356411, rs9348092 }\end{array}$ & 0.00084 & $\begin{array}{l}\text { LINC00473(n,d), LOC105378117(n\#), T(d), } \\
\text { MPC1(ce) }\end{array}$ \\
\hline 122 & rs2147866(*), rs6891675 & 0.00084 & CCDC192(n*), LINC01184(d) \\
\hline 123 & rs8178838(*) & 0.00088 & $\mathrm{APOH}\left(\mathrm{n}^{*}, \mathrm{~d}\right), \mathrm{CEP} 112(\mathrm{~d})$ \\
\hline 124 & rs13406850(*), rs10172452, rs1628975 & 0.00088 & $\mathrm{LRP} 1 \mathrm{~B}(\mathrm{~d})$ \\
\hline 125 & rs745247(*), rs7739748 & 0.00092 & CD83(d) \\
\hline 126 & rs6934819(*) & 0.00092 & ENPP3(d) \\
\hline 127 & rs7916162(*) & 0.00092 & TACC2(n*,d), PLEKHA1(ce) \\
\hline 128 & rs10764344(*), rs11013053 & 0.00092 & PIP4K2A(n*,d), PIP5K2A(ce) \\
\hline 129 & rs $3003177(*)$ & 0.00092 & ENSG00000223786(d\#) \\
\hline 130 & rs949719(*), rs1516651 & 0.00092 & $\operatorname{ATP} 10 B\left(n^{*}, d\right)$ \\
\hline 131 & rs938025(*) & 0.00096 & LINC00616(d), SLC7A11-AS1(d) \\
\hline 132 & rs11640395(*) & 0.00096 & ZFHX3(n*,d) \\
\hline 133 & rs2201369(*), rs10481102, rs13439041 & 0.00096 & BAALC(n*,d), BAALC-AS2(d) \\
\hline 134 & rs4521178(*) & 0.00096 & CPB1(n*,d), CPA3(d,ce) \\
\hline 135 & rs1400438(*), rs1516893 & 0.00096 & LINC01505(d) \\
\hline 136 & rs9864293(*) & 0.00096 & $\operatorname{IL1RAP}(\mathrm{d})$ \\
\hline 137 & rs2236570(*), rs613089 & 0.00096 & BCL9 $\left(n^{*}, d, c e\right), \operatorname{ACP} 6(d)$ \\
\hline 138 & rs10868152(*), rs7022329 & 0.00096 & $\operatorname{SLC} 28 \mathrm{~A} 3(\mathrm{n} *, \mathrm{~d})$ \\
\hline 139 & rs10501827(*) & 0.001 & SESN3(d) \\
\hline 140 & $\begin{array}{l}\text { rs8064765(*), rs11656652, } \\
\text { rs11079045, rs1032070, rs8069972, } \\
\text { rs2292755, rs4792992, rs4793253, } \\
\text { rs7224577 }\end{array}$ & 0.001 & $\begin{array}{l}\text { ATP6V0A1(n,d), CAVIN1(n,d), } \\
\text { LOC102725238(n\#), EZH1(d), CCR10(d), } \\
\text { PLEKHH3(d), RETREG3(n,d), MLX(n,d), } \\
\text { TUBG1(d), COASY(d,ce), CNTNAP1(d), } \\
\text { TUBG2(d), HSD17B1(d), NAGLU(d), } \\
\text { PSMC3IP(d), STAT3(ce), BECN1(ce) }\end{array}$ \\
\hline 141 & rs12750904(*) & 0.001 & $\mathrm{ABCD} 3(\mathrm{n} *, \mathrm{~d}, \mathrm{ce}), \mathrm{F} 3(\mathrm{~d})$ \\
\hline
\end{tabular}

Loci are ranked by $p$, which is the empirical $p$ for association of the index SNP with cardiovascular events. Reference SNP cluster IDs are reported for the index SNP (marked with an asterisk) and non-index clumped SNPs in the locus. For all SNPs in the locus, relevant genes were identified by proximity to the index or non-index SNPs (annotated with $\mathrm{n}$ in parenthesis, with an asterisk denoting genes in proximity to the index SNP). Additional relevant genes are reported based on cis and trans eQTLs (annotated with ce and te, respectively), and based on gene prioritization in DEPICT (annotated with d). Genes are reported by HGNC gene symbol if possible, or alternatively by Ensemble identifiers if no HGNC gene symbol exists.

$D E P I C T$ data-driven expression-prioritized integration for complex traits, eQTL expression quantitative trait loci, $H G N C$ human genome organisation gene nomenclature committee, SNP single nucleotide polymorphism.

Subsequent gene set enrichment analysis resulted in ten biological themes that highlight pathways that may be involved in the occurrence of CVD in chemotherapy-treated TC patients. These themes include the RAC2/RAC3 network, metabolism and adiposity, immune response, and caspase cascade/apoptosis.

Of special interest is the RAC2/RAC3 network that was identified as a prominent biological theme. Several recent reports link RAC2 and RAC3 to chemotherapy toxicity.
Rac2 deficiency protected Rac2-/- mice from bleomycininduced pulmonary fibrosis and resulted in lower mortality compared to wildtype mice [18]. In a rat model, differential Rac2 methylation was found in animals with acute lung injury induced by lipopolysaccharide compared to controls [19]. Although bleomycin-induced pneumonitis was not the endpoint of the current GWAS, it is a well-known side-effect of bleomycin in TC patients that originates from endothelial activation and is linked to endothelial 


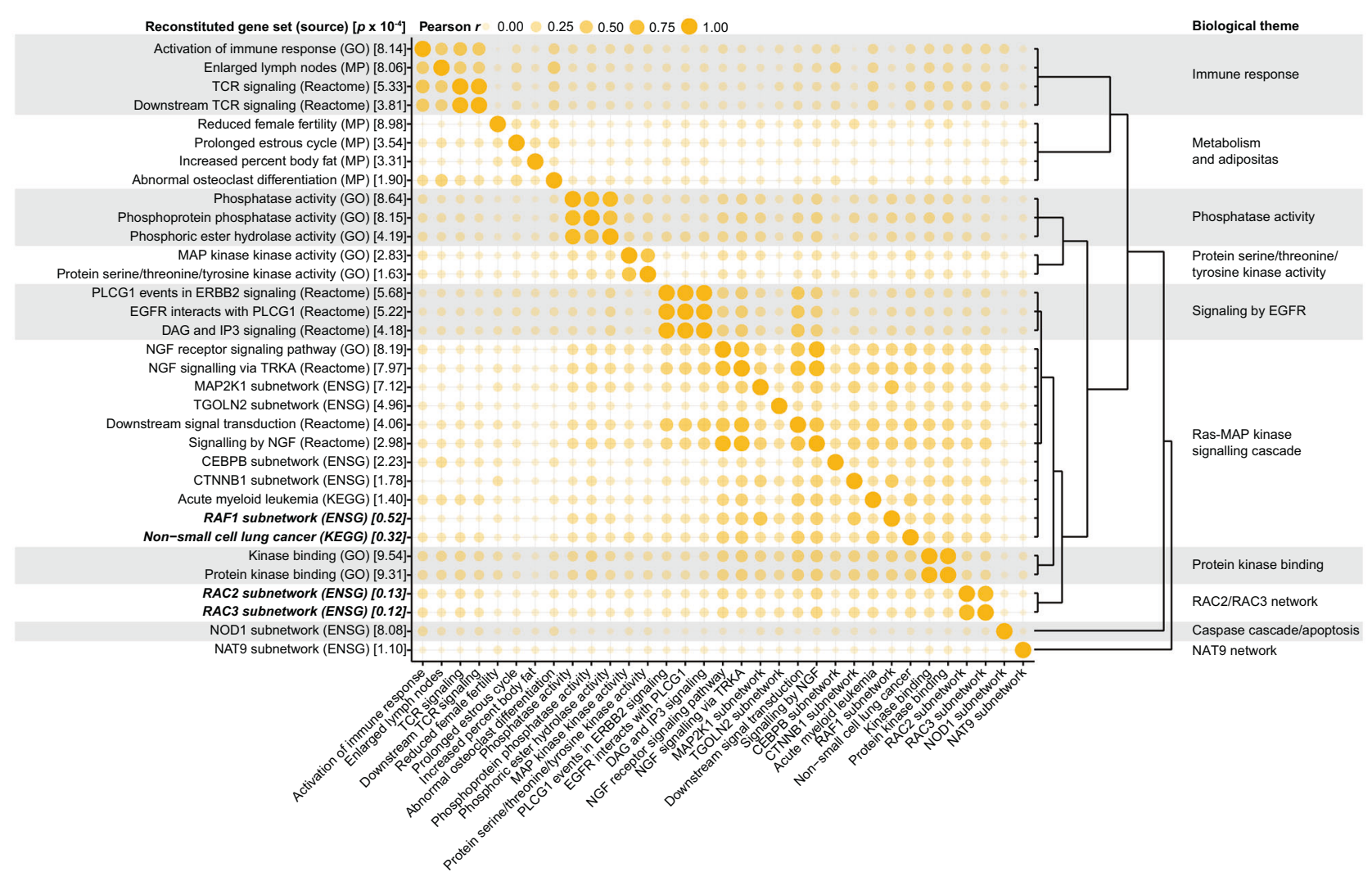

Fig. 2 The 33 gene sets that were enriched according to DEPICT were clustered into ten biological themes. The reconstituted gene sets were named after the predefined gene sets used in the development of DEPICT, and source databases are reported in brackets. The nominal enrichment $p$ for each reconstituted gene set as reported by DEPICT is reported in brackets, with emphasis added for gene sets with $p<1 \times 10^{-4}$. Clustering into biological themes was performed using affinity propagation clustering after calculating pairwise Pearson correlation between all enriched gene sets, as depicted in the bubble chart. CEBPB: CCAAT/enhancer binding protein beta, CTNNB1: catenin beta 1, DAG: diacylglycerol, EGFR: epidermal growth factor

dysfunction [20, 21]. Indeed, RAC2 has been implicated in endothelial activation and neovascularization, as well as leukocyte adhesion to the endothelial cell $[16,22]$. In an atherosclerosis model, Rac2 prevented plaque calcification by suppressing macrophage IL- $1 \beta$ expression. Furthermore, decreased RAC 2 expression and increased IL- $1 \beta$ expression were found in calcified coronary arteries from patients [23]. Rac1/2 pathways were involved in vascular injury in diabetic mice [24]. RAC3 has been suggested to inhibit senescence, and RAC3 expression is involved in the inflammatory response after TNF stimulation [25, 26]. Moreover, Rac3 modifies the induction of endothelial dysfunction by oxidized low-density lipoprotein in human umbilical vein endothelial cells [17]. Thus, the RAC2/ RAC3 network may play a role in atherosclerosis and senescence-pathophysiologic processes that have been implicated in the progress of CVD in TC patients-as well as bleomycin-induced pneumonitis. Interestingly, SNPs in receptor, ENSG: Ensembl gene, ERBB2: erb-b2 receptor tyrosine kinase 2, GO: gene ontology, IP3: inositol triphosphate, KEGG: Kyoto Encyclopedia of Genes and Genomes, MAP: mitogen-activated protein kinase, MAP2K1: MAP kinase kinase 1, MP: mammalian phenotype ontology, NAT9: N-acetyltransferase 9, NGF: nerve growth factor, NOD1: nucleotide binding oligomerization domain containing 1, PLCG1: phospholipase C, gamma 1, RAC2: ras-related C3 botulinum toxin substrate 2, RAC3: ras-related $\mathrm{C} 3$ botulinum toxin substrate 3, RAF1: Raf-1 proto-oncogene, serine/threonine kinase, TCR: $\mathrm{T}$ cell receptor; TGOLN2: trans-golgi network protein 2, TRKA: tropomyosin receptor kinase A.

RAC2 have also been found associated to cardiotoxicity due to anthracycline chemotherapy, suggesting that the RAC2/ RAC3 network is a common denominator in cardiovascular toxicity of multiple chemotherapeutic agents [27]. The occurrence of pathophysiological processes in which the RAC2/RAC3 network is involved, is illustrated in a cohort of $60 \mathrm{TC}$ patients with the observation that the number of CECs increases during consecutive cycles of platinumbased chemotherapy as sign of endothelial activation.

The biological themes of metabolism and adiposity, immune response, and caspase cascade/apoptosis are of particular interest, because these processes have been linked in literature to cardiovascular toxicity of platinum chemotherapy. The role of metabolism, adiposity, and endocrine dysfunction in the development of CVD has been well described in TC patients [28-30]. Besides this, the role of inflammation and immune response has been established in murine and cell models of platinum-induced nephrotoxicity, 


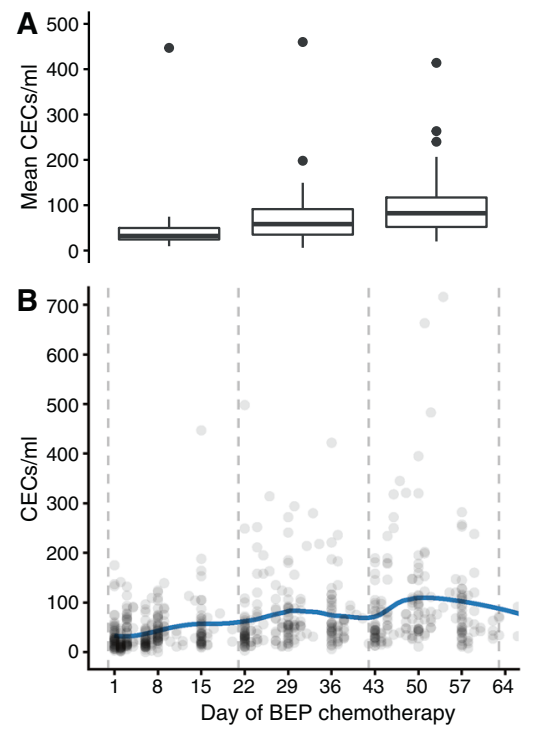

Fig. 3 The number of CECs rises during three cycles of BEP chemotherapy, based on measurements in 665 blood samples in 60 TC patients. Depicted are (a) the mean number of CECs per patient during each cycle, and (b) all CEC measurements with a LOESS curve. During the three consecutive cycles of chemotherapy the mean number of CECs per patient were median 32/ml (interquartile range, IQR 24-50), 58/ml (IQR 35-91), and 82/ml (20-117), respectively. Compared to the first cycle, the mean number of CECs was increased in the second and third cycle of chemotherapy (Wilcoxon signed rank test $p<0.001)$. BEP: bleomycin, etoposide, cisplatin, CEC: circulating endothelial cell.

evidenced by upregulation of TNF- $\alpha$ and a direct mediating role of $T$ lymphocytes [31-35]. The biological theme involving caspase activation and apoptosis is illustrated by murine and in vitro experiments indicating the role of caspase 1 and caspase 3 in platinum-induced nephrotoxicity, as well as endothelial cell apoptosis in response to cisplatin administration [20, 36, 37].

The low absolute incidence of TC and of CVD in chemotherapy-treated TC patients poses a challenge to perform meaningful GWAS on toxicity. Therefore, the current exploratory GWAS aimed to find relevant gene sets and possible biological themes rather than specific SNPs. To this end, the study was designed to minimize type II statistical errors whilst accepting a higher probability of type I statistical errors: in the trade-off between false positives and negatives, we avoided false negatives. Consequently, the current study should be regarded as hypothesisgenerating and the highlighted biological themes should be regarded as providing a promising base for future studies on genetic susceptibility and relevant biomarkers in CVD in TC patients. In this regard, results from the ongoing trials on genetic variation in TC survivors in relation to renal and cardiovascular toxicity (NCT02303015), as well as ototoxicity and neurotoxicity (NCT02890030, NCT02677727) are awaited.
The need to study SNPs associated with CVD in the specific population of TC patients derives from the notion that involved SNPs might differ from the currently known SNPs associated with increased risk of CVD in the general population. Indeed, only two of the 179 SNPs associated with CVD in the current GWAS were recorded to be associated with any cardiovascular phenotype in the NHGRI-EBI Catalog of published GWAS (catalog release 11 December 2017): rs2130392 and rs6773957 were associated with Kawasaki syndrome and adiponectin levels, respectively [38]. Nevertheless, future research should not only aim at exploring and validating results from genetic studies in TC cohorts, such as the biological themes highlighted in the current analysis, but also investigate the value of genetic risk scores derived from CVD GWAS in the general population.

The major strengths of this GWAS are the well-defined cohort, the completeness of follow-up, and the clinical relevance of addressing susceptibility to CVD in TC patients. Two additional remarks should be made on the study design. First, a broad definition of CVD (both venous and arterial) was used to define cases, because a stricter definition of only arterial events would unwarrantably compromise statistical power. Second, as we included patients treated from 1977 to 2011, follow-up duration varied widely, although selection bias in this regard may be considered unlikely given only a 1 year longer median follow-up duration for the cases compared to the control group, and equal ranges of follow-up duration for both groups.

\section{Conclusions}

In this exploratory GWAS, ten biological themes were linked with the occurrence of CVD in platinum-treated TC patients. These biological themes include metabolism and adipositas, immune response, apoptosis, and most prominently the RAC2/RAC3 network. This network has been implicated in bleomycin-induced lung injury, vascular oxidative stress, premature senescence, and endothelial activation. The biology of the RAC network was illustrated by observed CEC induction as sign of endothelial activation during consecutive courses of cisplatin-based chemotherapy in a TC cohort. Insight in the genetic variants determining susceptibility to CVD in TC patients can aid in the development of intervention strategies to prevent long-term sequelae of chemotherapy in often young cancer survivors.

\section{Compliance with ethical standards}

Conflict of interest The authors declare that they have no conflict of interest. 
Publisher's note Springer Nature remains neutral with regard to jurisdictional claims in published maps and institutional affiliations.

Open Access This article is licensed under a Creative Commons Attribution 4.0 International License, which permits use, sharing, adaptation, distribution and reproduction in any medium or format, as long as you give appropriate credit to the original author(s) and the source, provide a link to the Creative Commons license, and indicate if changes were made. The images or other third party material in this article are included in the article's Creative Commons license, unless indicated otherwise in a credit line to the material. If material is not included in the article's Creative Commons license and your intended use is not permitted by statutory regulation or exceeds the permitted use, you will need to obtain permission directly from the copyright holder. To view a copy of this license, visit http://creativecommons. org/licenses/by/4.0/.

\section{References}

1. Hanna NH, Einhorn LH. Testicular cancer - discoveries and updates. N Engl J Med. 2014;371:2005-16.

2. Haugnes HS, Wethal T, Aass N, Dahl O, Klepp O, Langberg CW, et al. Cardiovascular risk factors and morbidity in long-term survivors of testicular cancer: a 20-year follow-up study. J Clin Oncol. 2010;28:4649-57.

3. Fung C, Fossa SD, Milano MT, Sahasrabudhe DM, Peterson DR, Travis LB. Cardiovascular disease mortality after chemotherapy or surgery for testicular nonseminoma: a population-based study. J Clin Oncol. 2015;33:3105-15.

4. Oldenburg J, Kraggerud SM, Brydøy M, Cvancarova M, Lothe RA, Fossa SD. Association between long-term neuro-toxicities in testicular cancer survivors and polymorphisms in glutathione-stransferase-P1 and -M1, a retrospective cross sectional study. J Transl Med. 2007;5:70.

5. Oldenburg J, Kraggerud SM, Cvancarova M, Lothe RA, Fossa SD. Cisplatin-induced long-term hearing impairment is associated with specific glutathione s-transferase genotypes in testicular cancer survivors. J Clin Oncol. 2007;25:708-14.

6. Peters U, Preisler-Adams S, Hebeisen A, Hahn M, Seifert E, Lanvers C, et al. Glutathione S-transferase genetic polymorphisms and individual sensitivity to the ototoxic effect of cisplatin. Anticancer Drugs. 2000;11:639-43.

7. Boer H, Westerink NL, Altena R, Nuver J, Dijck-Brouwer DAJ, van Faassen M, et al. Single-nucleotide polymorphism in the 5- $\alpha$ reductase gene (SRD5A2) is associated with increased prevalence of metabolic syndrome in chemotherapy-treated testicular cancer survivors. Eur J Cancer. 2016;54:104-11.

8. Zaid MA, Gathirua-Mwangi WG, Fung C, Monahan PO, El-Charif O, Williams AM, et al. Clinical and genetic risk factors for adverse metabolic outcomes in North American testicular cancer survivors. J Natl Compr Canc Netw. 2018;16:257-65.

9. Drögemöller BI, Monzon JG, Bhavsar AP, Borrie AE, Brooks B, Wright GEB, et al. Association between SLC16A5 genetic variation and cisplatin-induced ototoxic effects in adult patients with testicular cancer. JAMA Oncol. 2017;92:414-7.

10. Wheeler HE, Gamazon ER, Frisina R, Perez-Cervantes C, El Charif O, Mapes B, et al. Variants in WFS1 and other Mendelian deafness genes are associated with cisplatin-associated ototoxicity. Clin Cancer Res. 2017;23:3325-33.

11. Purcell S, Neale B, Todd-Brown K, Thomas L, Ferreira MAR, Bender D, et al. PLINK: a tool set for whole-genome association and population-based linkage analyses. Am J Hum Genet. 2007; 81:559-75.

12. Westra HJ, Peters MJ, Esko T, Yaghootkar H, Schurmann C, Kettunen J, et al. Systematic identification of trans eQTLs as putative drivers of known disease associations. Nat Genet. 2013;45:1238-43.

13. Pers TH, Karjalainen JM, Chan Y, Westra HJ, Wood AR, Yang J, et al. Biological interpretation of genome-wide association studies using predicted gene functions. Nat Commun. 2015;6:5890.

14. Bodenhofer U, Kothmeier A, Hochreiter S. APCluster: an R package for affinity propagation clustering. Bioinformatics 2011;27:2463-64.

15. Strijbos MH, Rao C, Schmitz PI, Kraan J, Lamers CH, Sleijfer S, et al. Correlation between circulating endothelial cell counts and plasma thrombomodulin levels as markers for endothelial damage. Thromb Haemost. 2008;100:642-7.

16. De P, Peng Q, Traktuev DO, Li W, Yoden MC, March KL, et al. Expression of RAC2 in endothelial cells is required for the postnatal neovascular response. Exp Cell Res. 2009;315: 248-63.

17. He D, Xu L, Wu Y, Yuan Y, Wang Y, Liu Z, et al. Rac3, but not Rac1, promotes ox-LDL induced endothelial dysfunction by downregulating autophagy. J Cell Physiol. 2020;235:1531-42.

18. Arizmendi N, Puttagunta L, Chung KL, Davidson C, Rey-Parra J, Chao DV, et al. Rac2 is involved in bleomycin-induced lung inflammation leading to pulmonary fibrosis. Respir Res. 2014; 15:71.

19. Zhang XQ, Lv CJ, Liu XY, Hao D, Qin J, Tian HH, et al. Genomewide analysis of DNA methylation in rat lungs with lipopolysaccharide-induced acute lung injury. Mol Med Rep. 2013;7:1417-24.

20. Nuver J, De Haas EC, Van Zweeden M, Gietema JA, Meijer C. Vascular damage in testicular cancer patients: a study on endothelial activation by bleomycin and cisplatin in vitro. Oncol Rep. 2010;23:247-53.

21. Sleijfer S. Bleomycin-induced pneumonitis. Chest. 2001;120: 617-24.

22. Brenner B, Gulbins E, Busch GL, Koppenhoefer U, Lang F, Linderkamp O. L-selectin regulates actin polymerisation via activation of the small G-protein Rac2. Biochem Biophys Res Commun. 1997;231:802-7.

23. Ceneri N, Zhao L, Young BD, Healy A, Coskun S, Vasavada H, et al. Rac2 Modulates Atherosclerotic Calcification by Regulating Macrophage Interleukin-1 $\beta$ Production. Arterioscler Thromb Vasc Biol. 2017;37:328-40.

24. Bruder-Nascimento T, Callera GE, Montezano AC, He Y, Antunes TT, Cat AN, et al. Vascular injury in diabetic $\mathrm{db} / \mathrm{db}$ mice is ameliorated by atorvastatin: role of Rac1/2-sensitive Noxdependent pathways. Clin Sci. 2015;128:411-23.

25. Fernández Larrosa PN, Ruíz Grecco M, Mengual Gómez D, Alvarado CV, Panelo LC, Rubio MF, et al. RAC3 more than a nuclear receptor coactivator: a key inhibitor of senescence that is downregulated in aging. Cell Death Dis. 2015;6:e1902.

26. Alvarado CV, Rubio MF, Fernández Larrosa PN, Panelo LC, Azurmendi PJ, Ruiz Grecco M, et al. The levels of RAC3 expression are up regulated by TNF in the inflammatory response. FEBS Open Bio. 2014;4:450-7.

27. Tromp J, Steggink LC, Van Veldhuisen DJ, Gietema JA, van der Meer P. Cardio-oncology: progress in diagnosis and treatment of cardiac dysfunction. Clin Pharm Ther. 2017;101:481-90.

28. Haugnes HS, Aass N, Fosså SD, Dahl O, Klepp O, Wist EA, et al. Components of the metabolic syndrome in long-term survivors of testicular cancer. Ann Oncol. 2007;18:241-8.

29. Nuver J, Smit AJ, Postma A, Sleijfer DT, Gietema JA. The metabolic syndrome in long-term cancer survivors, an important target for secondary preventive measures. Cancer Treat Rev. 2002; 28:195-214.

30. Willemse PM, van der Meer RW, Burggraaf J, van Elderen SGC, de Kam ML, de Roos A, et al. Abdominal visceral and subcutaneous fat increase, insulin resistance and hyperlipidemia in 
testicular cancer patients treated with cisplatin-based chemotherapy. Acta Oncol. 2013;53:351-60.

31. Liu M, Chien C-C, Burne-Taney M, Molls RR, Racusen LC, Colvin $\mathrm{RB}$, et al. A pathophysiologic role for $\mathrm{T}$ lymphocytes in murine acute cisplatin nephrotoxicity. J Am Soc Nephrol. 2006;17:765-74.

32. Ramesh G, Reeves WB. TNF-alpha mediates chemokine and cytokine expression and renal injury in cisplatin nephrotoxicity. J Clin Investig. 2002;110:835-42.

33. Ramesh G, Reeves WB. TNFR2-mediated apoptosis and necrosis in cisplatin-induced acute renal failure. Am J Physiol Ren Physiol. 2003;285:F610-8.

34. Ramesh G, Reeves WB. p38 MAP kinase inhibition ameliorates cisplatin nephrotoxicity in mice. Am J Physiol Ren Physiol. 2005;289:F166-74.
35. Zhang B, Ramesh G, Norbury CC, Reeves WB. Cisplatin-induced nephrotoxicity is mediated by tumor necrosis factor-alpha produced by renal parenchymal cells. Kidney Int. 2007;72:37-44.

36. Faubel S, Ljubanovic D, Reznikov L, Somerset H, Dinarello CA, Edelstein CL. Caspase-1-deficient mice are protected against cisplatin-induced apoptosis and acute tubular necrosis. Kidney Int. 2004;66:2202-13.

37. Nagothu KK, Bhatt R, Kaushal GP, Portilla D. Fibrate prevents cisplatin-induced proximal tubule cell death. Kidney Int. 2005; 68:2680-93.

38. MacArthur J, Bowler E, Cerezo M, Gil L, Hall P, Hastings E, et al. The new NHGRI-EBI Catalog of published genome-wide association studies (GWAS Catalog). Nucleic Acids Res. 2017;45: D896-901. 